\title{
Laparoscopic salpingo-oophorectomy for ovarian torsion in the third trimester
}

\author{
David I. Shalowitz • J. Alejandro Rauh-Hain • \\ James Oyekan • Sarah L. Cohen
}

Received: 16 October 2012 / Accepted: 27 October 2012 /Published online: 9 November 2012

(C) Springer-Verlag Berlin Heidelberg 2012

Keywords Laparoscopy $\cdot$ Adnexal surgery $\cdot$ Pregnancy

\section{Introduction}

Ovarian torsion is uncommon in pregnancy, with incidence estimated between 1 and 10 per 10,000 pregnancies [1-3]. Furthermore, available case series estimate that ovarian torsion presents in the third trimester (i.e., after 28-weeks gestation) in $0-22 \%$ of cases $[2,4,5]$. In contrast to those who present in the first two trimesters, patients who present with concern for ovarian torsion in the third trimester may be more likely to undergo laparotomy rather than laparoscopy due to limited exposure allowed by the gravid uterus. [6] Upon literature review, we were able to identify only one report of laparoscopic salpingo-oophorectomy in the third trimester, though the details of the procedure were not provided [7]. We also identified case reports of thirdtrimester ovarian [8] and tubal [9] torsion treated laparoscopically without removal of adnexal structures. We herein report in detail and discuss a successful laparoscopic left salpingo-oophorectomy for ovarian torsion presenting in the 29th week of pregnancy.

\section{Case presentation}

A 30-year-old gravida 3 para 1 presented to our institution at 28 weeks, 5 days' gestation for evaluation of sudden-onset

D. I. Shalowitz · J. A. Rauh-Hain · J. Oyekan · S. L. Cohen

Department of Obstetrics and Gynecology,

Brigham and Women's Hospital,

Boston, MA, USA

D. I. Shalowitz $(\bowtie)$

75 Francis St. ASB1-3-078,

Boston, MA 02115, USA

e-mail: dshalowitz@partners.org left lower quadrant pain that woke her from sleep early in the morning. Aside from minimal nausea, a complete review of systems was negative. The patient had no prior surgical procedures; medical history was notable for depression, well-controlled on bupropion. On physical examination, the patient was afebrile with normal vital signs and marked tenderness over the left adnexa.

Sonographic evaluation of the pelvis revealed a $4.9 \times 2.2 \times$ 2.6-cm left ovary, containing a $2.7 \times 2.4 \times 1.9-\mathrm{cm}$ complex cystic mass. Assessment of arterial and venous Doppler flow to the ovary could not be performed due to overlying vascularity of the adnexal mass. The right ovary was unremarkable. Cervical length was $2.8 \mathrm{~cm}$, there was no sonographic evidence of abruption, and the fetal portion of the exam was within normal limits with biophysical profile 8 points out of 8 . Based on clinical and laboratory findings, ovarian torsion was felt to be more likely than other causes of acute lower abdominal pain in this patient, e.g., appendicitis, nephrolithiasis, placental abruption, or preterm contractions.

Obstetrics and anesthesiology consultations were obtained with plan made for diagnostic laparoscopy and possible ovarian detorsion versus salpingo-oophorectomy. She was positioned in the left lateral decubitus position and continuous fetal heart rate monitoring was initiated. Equipment for emergency cesarean delivery and neonatal resuscitation was immediately available. Rapid-sequence induction of anesthesia with endotracheal intubation was performed. An orogastric tube was placed to decompress the stomach. The patient received appropriate preoperative antibiotic and venous thromboembolism prophylaxis.

After standard preparation and draping of the abdomen, the left costal margin and the uterine fundus were outlined to assist with port placement. A 5-mm incision was made just below the inferior costal margin on the left, in the midclavicular line. A 5-mm port was inserted into the peritoneal cavity under direct laparoscopic vision and, after abdominal access was visually confirmed, the abdomen was insufflated to $15 \mathrm{~mm} \mathrm{Hg}$ with carbon dioxide gas. The patient was then 
tilted to the right to facilitate movement of the uterus away from the affected adnexa.

On survey of the abdomen, the left ovary was torsed three times around its vascular pedicle and appeared necrotic. A spinal needle was then used to assess prospective accessory trocar placement sites for adequate visibility and distance from the adnexa and uterus. An additional 5-mm port was placed under direct visualization in the left upper quadrant approximately $8 \mathrm{~cm}$ below and lateral to the initial port site, along with a third $5-\mathrm{mm}$ port in the left upper quadrant near the midline (Fig. 1). The ovary was then detorsed with a blunt grasper and observed for $5 \mathrm{~min}$. The necrotic appearance of the ovary did not change, and the decision was made to proceed with salpingo-oophorectomy. The initial left upper quadrant trocar was then extended to $12 \mathrm{~mm}$ to assist with specimen retrieval.

The LigaSure device (Covidien, Mansfield, MA) was used to perform a left salpingo-oophorectomy in standard fashion. Blood loss was minimal. The fallopian tube and ovary were removed from the abdomen without spillage using the 10-mm EndoCatch (Covidien, Mansfield, MA) retrieval bag and sent for permanent section. The abdomen was desufflated and the port sites closed in the usual manner. Fetal heart rate during the entire procedure was between 110 and $120 \mathrm{bpm}$ with minimal variability consistent with general anesthesia.

The patient was brought to the Labor and Delivery Unit for postoperative monitoring. She was discharged without complication on postoperative day2. Final pathology showed a mature, cystic teratoma, as well as hemorrhagic fat necrosis, consistent with ovarian torsion.

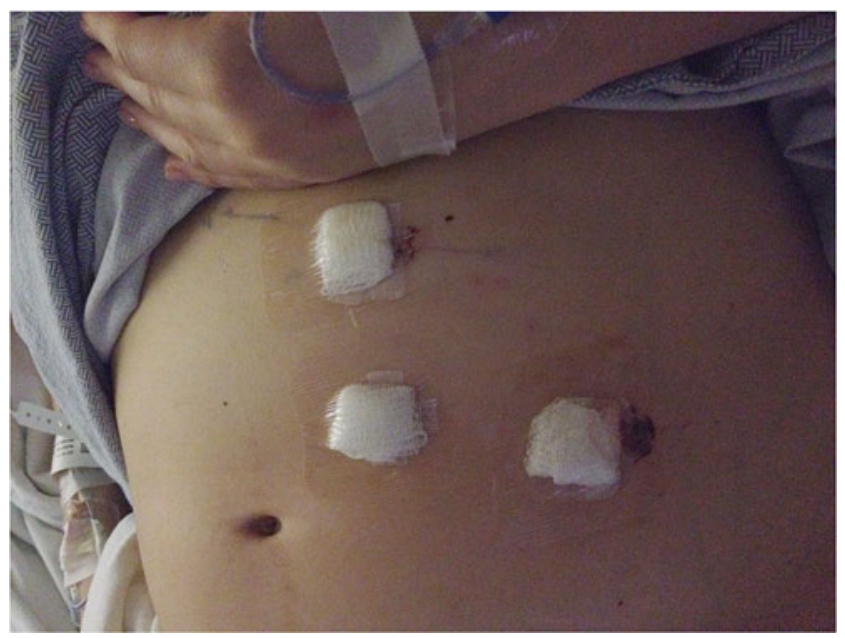

Fig. 1 Port placement. Note: line visible above superior-most port represents the costal margin

\section{Discussion}

Ovarian torsion in the third trimester is uncommon, and laparoscopic treatment of torsion at this stage of pregnancy requires the surgical team to address fetal, maternal, and technical aspects of this procedure. First, we recommend intraoperative assessment of fetal heart rate with obstetrics and neonatology consultations obtained prior to surgery to ensure that the appropriate resources are available should emergency cesarean delivery become necessary. Postoperatively, the patient should be monitored per institutional protocol for laparoscopic surgery and cardiotocography should be utilized to screen for fetal distress and preterm labor prior to discharge.

Second, standard anesthesia procedures for laparoscopic surgery in a pregnant patient should be utilized, including rapid-sequence induction and intubation, gastric decompression, and avoidance of hypercapnia. Moreover, careful attention should be paid to lateral displacement of the gravid uterus away from the vena cava as there is a risk of decreased venous return beyond that expected from pneumoperitoneum alone. In this case report, torsion of the left ovary required the patient to be in the supine position during the salpingo-oophorectomy; time spent without leftward tilt was minimized. In addition, given the importance of minimizing maternal and fetal exposure to surgical anesthesia, the procedure was completed laparoscopically only because we felt that we could use this approach safely and expeditiously. As mentioned above, equipment was immediately available for conversion to laparotomy.

Finally, when considering laparoscopic assessment for and treatment of ovarian torsion in the third trimester, we recommend conscientious placement of trocars to avoid vascular or uterine injury from the insufflation needle. In this case, direct left upper quadrant subcostal port placement under visualization was sufficient to allow for adequate operating space and avoidance of organ injury. Conversion to an open approach may be necessary if visualization remains limited. Salpingo-oophorectomy may be considered if there is any concern for ovarian necrosis after initial detorsion given the theoretical possibility of adverse obstetric sequelae (e.g., preterm labor) if there is subsequent inflammation or infection of the adnexa

\section{Conclusion}

In summary, although uncommon, ovarian torsion in the third trimester may be treated laparoscopically in selected cases with appropriate technical modifications and interdisciplinary consultation. 
Declaration of interest The authors report no conflicts of interest. The authors alone are responsible for the content and writing of the paper.

\section{References}

1. Hasson J, Tsafrir Z, Azem F, Bar-On S, Almog B, Mashiach R et al (2010) Comparison of adnexal torsion between pregnant and nonpregnant women. Am J Obstet Gynecol 202:536, e1-6

2. Smorgick N, Pansky M, Feingold M, Herman A, Halperin R, Maymon R (2009) The clinical characteristics and sonographic findings of maternal ovarian torsion in pregnancy. Fertil Steril 92 (6):1983-1987, Epub 2008 Nov 5

3. Hasiakos D, Papakonstantinou K, Kontoravdis A, Gogas L, Aravantinos L, Vitoratos N (2008) Adnexal torsion during pregnancy: report of four cases and review of the literature. J Obstet Gynaecol Res 34(4 Pt 2):683-687

4. Hibbard LT (1985) Adnexal torsion. Am J Obstet Gynecol 152:456-461

5. Balci O, Gezginc K, Karatayli R, Acar A, Celik C, Colakoglu MC (2008) Management and outcomes of adnexal masses during pregnancy: a 6-year experience. J Obstet Gynaecol Res 34(4):524-528

6. Chang SD, Yen CF, Lo LM, Lee CL, Liang CC (2011) Surgical intervention for maternal ovarian torsion in pregnancy. Taiwan $\mathrm{J}$ Obstet Gynecol 50(4):458-462

7. Upadhyay A, Stanten S, Kazantsev G, Horoupian R, Stanten A (2007) Laparoscopic management of a nonobstetric emergency in the third trimester of pregnancy. Surg Endosc 21:1344-1348

8. Roman H, Diguet A, Loisel C, Resch B, Marpeau L, Taleb F (2008) Laparoscopic management of adnexal mass during the third trimester of pregnancy. J Minim Invasive Gynecol 15:S1eS156

9. Chohan L, Ramirez MM, Wray CJ, Kilpatrick CC (2011) Laparoscopic management of fallopian tube torsion at 35 weeks of gestation: case report. J Minim Invasive Gynecol 18(3):390-392 